\title{
The Effects of Socioeconomic Status, Parental Education Level, Oral and Dental Health Practices, Dietary Habits and Anthropometric Measurements on Dental Health in 12-Year-Old School Children
}

Jiyan ASLAN CEYLAN ( $\square$ jynaslan@gmail.com )

Mardin Artuklu University: T C Mardin Artuklu Universitesi https://orcid.org/0000-0003-1649-3586

YUSUF ASLAN

T C Mardin Artuklu Universitesi

AYSE OZFER OZCELIKK

Ankara University: Ankara Universitesi

\section{Research}

Keywords: anthropometry, diet, DMFT, tooth, socioeconomic

Posted Date: August 2nd, 2021

DOl: https://doi.org/10.21203/rs.3.rs-713036/v1

License: (c) (i) This work is licensed under a Creative Commons Attribution 4.0 International License.

Read Full License 


\section{Abstract \\ Background}

Dental caries is the most common progressive chronic disease in school-age children with an increasing prevalence as children grow up. This study aims to examine the effects of socioeconomic status, parental education level, oral and dental health practices, dietary habits and anthropometric measurements on dental health in 12-year-old schoolchildren.

\section{Methods}

The sample of the study consisted of 254 children ( $44.1 \%$ boys and $55.9 \%$ girls) in three schools, which were identified as low, moderate and high socioeconomic status.

\section{Results}

It was found that $70.9 \%$ of the children have dental caries on their permanent teeth. The frequency of seeing a dentist, the status of receiving oral and dental health education and the frequency of changing toothbrush vary according to the socioeconomic status. Oral and dental health indicators were determined to be affected by the frequency and duration of tooth brushing. It was found that dmft values of the children consuming molasses and table sugar are higher. There is a negative correlation between oral and dental health indicators and anthropometric measurements and parental education level.

\section{Conclusions}

Dietary habits, anthropometric measurements, oral and dental health practices, gender, and parental socioeconomic status and education level were shown to be effective on caries risk.

\section{Background}

Oral and dental health is an integral part of complete health and well-being (Hafez, 2017). In most developing low-income countries, the prevalence of dental caries is high and more than $90 \%$ of caries are reported to be untreated (Moynihan and Petersen, 2004). Dental caries is the most common progressive chronic disease in school-age children with an increasing prevalence as children grow up (Edelstein, 2002; Koksal et al., 2011). Recent trends relating to the increase in the prevalence of dental caries in children emphasize the need for more comprehensive measures as it is a preventable disease (Touger-Decker and Mobley, 2013). Oral and dental health in school-age children totally depends on oral hygiene behavior of children and their parents, dietary habits, parental education level, socioeconomic status, regular dental examination, adequate fluoride supplementation, oral microflora, age and other demographic and cultural characteristics (Koposova et al., 2010; Koksal et al., 2011; Bafti et al., 2015). 
Caries risk assessment and determining leading risk factors enable effective prevention programs to be implemented at different levels (families, schools, institutions, local communities, etc.). In assessing a caries risk, a single method or model cannot simultaneously measure host resistance, microbial pathogens and carcinogenicity of the diet. Therefore, caries risk should be assessed by analyzing and integrating several causal factors (Amila et al., 2007). This study aims to examine the effects of socioeconomic status, parental education level, oral and dental health practices, dietary habits and anthropometric measurements on dental health in 12-year-old school children, and unlike the other studies, many factors involved in the etiology of dental caries were assessed together.

\section{Materials And Methods}

This is a descriptive study whose sample was composed of a total of 254 voluntary 12-year-old school children ( $44.1 \%$ boys and $55.9 \%$ girls) enrolled in three different middle schools (low, moderate and high socioeconomic level) in Mardin-Turkey. The World Health Organization develops basic methods and criteria for use in oral and dental health field surveys and recommends the use of specific age or age bands to make comparisons between countries. It is stated in the "Basic Principles" of the World Health Organization that it is sufficient to select 5 years of age to determine the condition of milk teeth and to select sample among 12- and 15-year-old children who have different risks to determine the condition of permanent teeth in childhood. This study was carried out with 12-year-old children due to the fact that all permanent teeth except for the third molars should erupt until 12 years of age and this age group is a global indicator age group for monitoring international comparisons and disease trends (WHO, 2013). The study was conducted with the approval of the Ethics Committee of Mardin Artuklu University dated 11.01.2018 and no. 2018/01-3.

The data were collected by face-to-face interviews via a questionnaire form including socioeconomic status, parental education level, oral and dental health practices, and anthropometric measurement. Children's height $(\mathrm{cm})$, body weight $(\mathrm{kg})$, waist circumference $(\mathrm{cm})$, hip circumference $(\mathrm{cm})$, upper middle arm circumference $(\mathrm{cm})$, triceps skinfold thickness $(\mathrm{mm})$ and biceps skinfold thickness $(\mathrm{mm})$, were taken in accordance with the technique. Waist circumference $\geq 84.5 \mathrm{~cm}$ for boys and $\geq 81.9 \mathrm{~cm}$ for girls were considered "risk", but the ones below these values were considered "normal" (Cruz and Goran, 2004). Waist-to-height ratios were classified as "take care" if $<0.4$, "normal" if $0.4-0.5$, "take care" if $0.5-0.6$, and "take action" if $\geq 0.6$ (Ashwell and Hsieh, 2005). Body Mass Index ( $\mathrm{kg} \backslash \mathrm{m} 2)$ was classified as $<3$ "too weak", $\geq 3-<15$ "weak", $\geq 15-<85$ "normal", $\geq 85-<97$ "overweight", and $\geq 97$ "obese" according to the 12year-old table of percentiles (WHO, 2007).

Clinical examinations were performed by a dentist to assess the oral and dental health of the children. The dentist determined the number of teeth affected by caries and its results for each child and marked them in the oral examination form. The sums of the number of decayed teeth (DT), missing teeth (MT) and filled teeth (FT) (Decayed, Missing, and Filled Teeth: DMFT), and teeth surfaces (Decayed, Missing, and Filled Surfaces: DMFS) were calculated. DMFT and dmfs indices of the children were determined as a result of these calculations. $\mathrm{dmft}$ and DMFS indices were used for milk teeth. The missing teeth were 
not included in the examination for milk teeth. The dental caries levels of the children were determined using WHO classification based on the means DMFT and dmft (< 1.2 "very low", 1.2-2.6 "low", 2.7-4.4 "moderate", 4.5-6.5 "high", and > 6.5 "very high") (WHO, 2013).

SPSS (Statistical Package for the Social Sciences) package program was used to analyze the data. ChiSquare and Fisher's Exact Chi-Square tests were performed to determine whether there was a significant relationship between qualitative variables. Spearman Correlation was used to determine the relationship between the factors affecting oral and dental health indicators. Statistical significance was evaluated at $p<0.01$ and $p<0.05$. The confidence interval for all statistical tests was adopted as $95.0 \%$.

\section{Results}

A total of 25412 -year-old children (44.1\% boys and $55.9 \%$ girls) participated in the study. It was found that $70.9 \%$ of the children have dental caries on their permanent teeth and $44.1 \%$ of them have at least one caries on their milk teeth. Moreover, it was found that the number of girls who have caries on their permanent teeth and boys who have caries on their milk teeth is higher $(p<0.05)$. dmft and dmfs indices were found to be very low in $47.2 \%$ and $75.2 \%$ of the children, respectively. It was discovered that low DMFT rates (girls $20.5 \%$, boys $9.8 \%$ ) were higher in girls and high dmfs rates (girls $2.1 \%$, male, $9.8 \%$ ) were higher in boys $(p<0.05)($ Table 1$)$. 
Table 1

Classification of indicators of permanent and milk teeth of children

\begin{tabular}{|c|c|c|c|c|c|c|c|}
\hline \multirow{2}{*}{$\begin{array}{l}\text { Oral health indicators } \\
\text { Permanent teeth }\end{array}$} & \multicolumn{2}{|l|}{ Boy } & \multicolumn{2}{|l|}{ Girl } & \multicolumn{2}{|c|}{ Total } & \multirow{2}{*}{$\begin{array}{l}\text { Chi-Square test } \\
\mathrm{p}\end{array}$} \\
\hline & $\mathrm{n}$ & $\%$ & $\mathrm{n}$ & $\%$ & $\mathbf{n}$ & $\%$ & \\
\hline Have caries & 69 & 61.6 & 111 & 78.2 & 180 & 70.9 & 8.319 \\
\hline Have not caries & 43 & 38.4 & 31 & 21.8 & 74 & 29.1 & $0.004^{*}$ \\
\hline \multicolumn{8}{|l|}{ Milk teeth } \\
\hline Have caries & 59 & 52.7 & 53 & 37.3 & 112 & 44.1 & 5.988 \\
\hline Have not caries & 53 & 47.3 & 89 & 62.7 & 142 & 55.9 & $0.014^{*}$ \\
\hline \multicolumn{8}{|l|}{ Classification of DMFT } \\
\hline Very low & 63 & 56.2 & 57 & 40.1 & 120 & 47.2 & \multirow{5}{*}{$\begin{array}{l}12.561 \\
0.014^{\star}\end{array}$} \\
\hline Low & 11 & 9.8 & 29 & 20.5 & 40 & 15.7 & \\
\hline Middle & 25 & 22.4 & 47 & 33.1 & 72 & 28.3 & \\
\hline High & 11 & 9.8 & 8 & 5.6 & 19 & 7.5 & \\
\hline Very high & 2 & 1.8 & 1 & 0.7 & 3 & 1.3 & \\
\hline \multicolumn{7}{|l|}{ Classification of dmfs } & \multirow{7}{*}{$\begin{array}{l}14.698 \\
0.005^{\star}\end{array}$} \\
\hline Very low & 73 & 65.2 & 118 & 83.2 & 191 & 75.2 & \\
\hline Low & 17 & 15.2 & 10 & 7.0 & 27 & 10.6 & \\
\hline Middle & 10 & 8.9 & 11 & 7.7 & 21 & 8.3 & \\
\hline High & 11 & 9.8 & 3 & 2.1 & 14 & 5.5 & \\
\hline Very high & 1 & 0.9 & - & - & 1 & 0.4 & \\
\hline Total & 112 & 100.0 & 142 & 100.0 & 254 & 100.0 & \\
\hline
\end{tabular}

It was found that nearly all of the children brush their teeth $(96.1 \%)$ and the number of those who do not brush their teeth $(7.9 \%)$ is higher among ones the with low socioeconomic status $(p<0.05)$. $34.3 \%$ of the students reported that they brush their teeth once a day and $21.7 \%$ reported they sometimes brush their teeth. The number of those who brush their teeth several times a week $(10.1 \%)$ is higher among the ones with moderate socioeconomic status and the number of those who brush their teeth three times a day $(6.3 \%)$ is higher among the ones with high socioeconomic status $(p<0.05)$. Nearly half of the children (49.2\%) stated that they first saw a dentist at the age of $6-10$ and $14.2 \%$ reported that they have never seen a dentist. It was found that the number of those who see a dentist 1-2 times a year (30.0\%), have previously received oral and dental health education (65.6\%) and change their toothbrush every three months $(43.8 \%)$ is higher among the ones with high socioeconomic status $(p<0.05)$. It was discovered 
that $39.0 \%$ of children have harmful oral and dental health habits in which lip bite (62.6\%) is the leading (Table 2). 
Table 2

Information on oral and dental health of children

\begin{tabular}{|c|c|c|c|c|c|c|c|c|c|}
\hline \multirow[t]{2}{*}{ Information } & \multicolumn{2}{|c|}{$\begin{array}{l}\text { Low } \\
\text { socioeconomic } \\
\text { status }\end{array}$} & \multicolumn{2}{|c|}{$\begin{array}{l}\text { Moderate } \\
\text { socioeconomic } \\
\text { status }\end{array}$} & \multicolumn{2}{|c|}{$\begin{array}{l}\text { High } \\
\text { socioeconomic } \\
\text { status }\end{array}$} & \multicolumn{2}{|c|}{ Total } & \multirow[t]{2}{*}{$\begin{array}{l}\text { Statistics } \\
\mathrm{p}\end{array}$} \\
\hline & $\mathbf{n}$ & $\%$ & $\mathbf{n}$ & $\%$ & $\mathrm{n}$ & $\%$ & $n$ & $\%$ & \\
\hline \multicolumn{9}{|c|}{ Status brushing teeth } & $5.573^{\star}$ \\
\hline Brushes & 82 & 92.1 & 100 & 99.0 & 62 & 96.9 & 244 & 96.1 & $0.049 * \star \star$ \\
\hline Not brushes & 7 & 7.9 & 1 & 1.0 & 2 & 3.1 & 10 & 3.9 & \\
\hline \multicolumn{9}{|c|}{ The frequency of tooth brushing } & $21.932 *$ \\
\hline $\begin{array}{l}1 \text { time per } \\
\text { day }\end{array}$ & 22 & 24.7 & 37 & 36.6 & 28 & 43.8 & 87 & 34.3 & $0.010 * \star \star$ \\
\hline 2 times a day & 29 & 32.6 & 24 & 23.8 & 21 & 32.8 & 74 & 29.1 & \\
\hline $\begin{array}{l}3 \text { times per } \\
\text { day }\end{array}$ & 4 & 4.5 & 2 & 2.0 & 4 & 6.3 & 10 & 3.9 & \\
\hline $\begin{array}{l}\text { Several times } \\
\text { a week }\end{array}$ & 5 & 5.6 & 11 & 10.9 & 2 & 3.1 & 18 & 7.1 & \\
\hline Sometimes & 22 & 24.7 & 26 & 25.7 & 7 & 10.9 & 55 & 21.7 & \\
\hline Never & 7 & 7.9 & 1 & 1.0 & 2 & 3.1 & 10 & 3.9 & \\
\hline \multicolumn{9}{|c|}{ The frequency of brush replacement } & $49.286^{\star \star}$ \\
\hline Quarterly & 21 & 23.6 & 10 & 9.9 & 28 & 43.8 & 59 & 23.2 & $0.000 * \star \star$ \\
\hline $\begin{array}{l}\text { Once in a six } \\
\text { month }\end{array}$ & 19 & 21.3 & 30 & 29.7 & 24 & 37.5 & 73 & 28.8 & \\
\hline Once a year & 34 & 38.2 & 54 & 53.5 & 9 & 14.0 & 99 & 39.0 & \\
\hline $\begin{array}{l}\text { Once in two } \\
\text { years }\end{array}$ & 8 & 9.0 & 6 & 5.9 & 1 & 1.6 & 15 & 5.9 & \\
\hline Never & 7 & 7.9 & 1 & 1.0 & 2 & 3.1 & 8 & 3.1 & \\
\hline \multicolumn{9}{|c|}{ The age of see the dentist for the first time (years) } & $16.930 * *$ \\
\hline & & & & & & & & & $0.010 * \star \star$ \\
\hline \multicolumn{10}{|c|}{ * Fisher's Exact Test } \\
\hline \multicolumn{10}{|c|}{ ** Chi-Square test } \\
\hline \multicolumn{10}{|l|}{$* * * p<0.05$} \\
\hline
\end{tabular}




\begin{tabular}{|c|c|c|c|c|c|c|c|c|c|}
\hline $\begin{array}{l}1-5 \text { years } \\
\text { old }\end{array}$ & 16 & 18.0 & 20 & 19.7 & 25 & 39.1 & 61 & 24.0 & \\
\hline $\begin{array}{l}6-10 \text { years } \\
\text { old }\end{array}$ & 40 & 44.9 & 55 & 54.5 & 30 & 46.9 & 125 & 49.2 & \\
\hline $\begin{array}{l}11-12 \text { years } \\
\text { old }\end{array}$ & 16 & 18.0 & 11 & 10.9 & 5 & 7.8 & 32 & 12.6 & \\
\hline Never & 17 & 19.1 & 15 & 14.9 & 4 & 6.2 & 36 & 14.2 & \\
\hline \multicolumn{9}{|c|}{ The frequency of see the dentist $(n=218)$} & $17.783 * \star$ \\
\hline $\begin{array}{l}\text { When } \\
\text { complaints }\end{array}$ & 64 & 88.9 & 77 & 89.5 & 42 & 70.0 & 183 & 83.9 & $0.001 * \star \star$ \\
\hline $\begin{array}{l}1-2 \text { times } \\
\text { per year }\end{array}$ & 8 & 11.1 & 9 & 10.5 & 18 & 30.0 & 35 & 16.1 & \\
\hline \multicolumn{10}{|c|}{ Previously oral health education } \\
\hline Have training & 18 & 20.2 & 19 & 18.8 & 42 & 65.6 & 79 & 31.1 & $47.629 * \star$ \\
\hline $\begin{array}{l}\text { Have not } \\
\text { training }\end{array}$ & 71 & 79.8 & 82 & 81.2 & 22 & 34.4 & 175 & 68.9 & 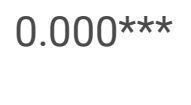 \\
\hline \multicolumn{9}{|c|}{ Harmful oral health habits } & $2.923^{\star \star}$ \\
\hline Have not & 48 & 53.9 & 65 & 64.4 & 42 & 65.6 & 155 & 61.0 & 0.232 \\
\hline Have & 41 & 46.1 & 36 & 35.6 & 22 & 34.4 & 99 & 39.0 & \\
\hline Total & 89 & 100.0 & 101 & 100.0 & 64 & 100.0 & 254 & 100 & \\
\hline \multicolumn{10}{|c|}{ If there are harmful oral habits $(n=41)^{x}$} \\
\hline Lip biting & 29 & 70.7 & 20 & 55.5 & 22 & 100.0 & 62 & 62.6 & \\
\hline Nail biting & 15 & 36.6 & 20 & 55.5 & 15 & 68.2 & 50 & 50.5 & \\
\hline Cheek Biting & 7 & 17.1 & 5 & 13.9 & 1 & 4.5 & 13 & 13.1 & \\
\hline \multicolumn{10}{|c|}{ * Fisher's Exact Test } \\
\hline \multicolumn{10}{|c|}{ ** Chi-Square test } \\
\hline \multicolumn{10}{|l|}{$\star \star \star p<0.05$} \\
\hline x Multiple ans & s w & ven & & & & & & & \\
\hline
\end{tabular}

There are $1.7 \pm 1.78$ decay, $0.1 \pm 0.35$ missing and $0.2 \pm 0.69$ fillings in permanent teeth of the participants. The mean numbers of decayed and filled milk teeth are $1.0 \pm 1.49$ and $0.5 \pm 0.36$, respectively (data not shown). The mean DMFT is $2.0 \pm 1.90$, and dmft is $1.0 \pm 1.57$. dt, dmft, ds and dmfs values of boys were found to be higher than of girls $(p<0.05)$. It was determined that those who brush 
their teeth, brush their teeth after the meal and before bedtime, brush in a circular style and change their toothbrush every three months have better oral examinations $(p>0.05)$. Those who brush their teeth three times a day (DMFT 1.3 \pm 1.42 , dmfs $0.0 \pm 0.00)$ have better permanent $(p>0.05)$ and milk teeth $(p<0.05)$ examination than sometimes brushers (DMFT $2.4 \pm 2.02$, dmfs $1.5 \pm 1.93$ ). According to the brushing duration, those who brush their teeth for $2-3 \mathrm{~min}$. have the minimum mean DMFT values $(1.8 \pm 1.70)(p>$ $0.05)$ and it was found that those who brush more than three min. $(0.2 \pm 0.58)$ have lower mean $\mathrm{dmfs}$ values than those who do not know their brushing duration $(1.9 \pm 1.20)(p<0.05)($ Table 3$)$. 
Table 3

Oral health indicators according to gender and oral/dental health practices

\begin{tabular}{|c|c|c|c|c|c|}
\hline \multirow[t]{4}{*}{ Habits } & \multicolumn{2}{|c|}{ Permanent teeth } & \multicolumn{2}{|l|}{ Milk teeth } & \multirow[t]{4}{*}{$\mathbf{p}$} \\
\hline & \multicolumn{2}{|c|}{$\mathrm{DMFT}^{\mathrm{c}}$} & \multicolumn{2}{|l|}{$\mathrm{dmft}^{\mathrm{d}}$} & \\
\hline & $\mathrm{X} \pm \mathrm{SS}$ & Median & $\mathrm{X} \pm \mathrm{SS}$ & Median & \\
\hline & & (Min-Max) & & (Min-Max) & \\
\hline \multicolumn{6}{|l|}{ Gender } \\
\hline \multicolumn{5}{|c|}{ Boy $1.9 \pm 2.091 .00(0.00-9.00) 1.4 \pm 1.831 .00(0.00-9.00)$} & $0.073^{c}$ \\
\hline \multicolumn{5}{|c|}{ Girl $2.1 \pm 1.732 .00(0.00-9.00) 0.7 \pm 1.260 .00(0.00-6.00)$} & $0.003^{\mathrm{d} *}$ \\
\hline \multicolumn{6}{|c|}{ Total $2.0 \pm 1.902 .00(0.00-9.00) 1.0 \pm 1.570 .00(0.00-9.00)$} \\
\hline \multicolumn{6}{|l|}{ Status brushing teeth ${ }^{a}$} \\
\hline Brushes & $\begin{array}{l}2.0 \pm \\
1.90\end{array}$ & $\begin{array}{l}2.00(00- \\
9.00)\end{array}$ & $0.9 \pm 1.54$ & $\begin{array}{l}0.00(00- \\
9.00)\end{array}$ & \multirow{2}{*}{$\begin{array}{l}0.675^{\mathrm{c}} \\
0.450^{\mathrm{d}}\end{array}$} \\
\hline Not brushes & $\begin{array}{l}2.2 \pm \\
1.81\end{array}$ & $\begin{array}{l}2.00(00- \\
5.00)\end{array}$ & $1.6 \pm 2.22$ & $\begin{array}{l}0.50(00- \\
6.00)\end{array}$ & \\
\hline \multicolumn{5}{|l|}{ Tooth brushing time ${ }^{b}$} & $0.099^{c}$ \\
\hline After meals & $\begin{array}{l}2.4 \pm \\
1.79\end{array}$ & $\begin{array}{l}2.00(00- \\
6.00)\end{array}$ & $0.8 \pm 1.64$ & $\begin{array}{l}0.00(00- \\
9.00)\end{array}$ & \multirow[t]{4}{*}{$0.150^{d}$} \\
\hline $\begin{array}{l}\text { After meals and before } \\
\text { bedtime }\end{array}$ & $\begin{array}{l}1.5 \pm \\
1.69\end{array}$ & $\begin{array}{l}1.00(00- \\
6.00)\end{array}$ & $0.5 \pm 0.98$ & $\begin{array}{l}0.00(00- \\
4.00)\end{array}$ & \\
\hline Before bedtime & $\begin{array}{l}2.0 \pm \\
1.96\end{array}$ & $\begin{array}{l}2.00(00- \\
9.00)\end{array}$ & $1.2 \pm 1.68$ & $\begin{array}{l}0.50(00- \\
6.00)\end{array}$ & \\
\hline Uncertain & $\begin{array}{l}2.2 \pm \\
2.00\end{array}$ & $\begin{array}{l}2.00(00- \\
9.00)\end{array}$ & $1.6 \pm 2.22$ & $\begin{array}{l}0.00(00- \\
6.00)\end{array}$ & \\
\hline
\end{tabular}

\section{aMann-Whitney U Test}

${ }^{\text {b} K r u s k a l ~ W a l l i s ~ T e s t ~}$

${ }^{c} \mathrm{p}$ value for DMFT

${ }^{d} p$ value for $p \mathrm{dmft}$

${ }^{*} \mathrm{p}<0.05$

$x . x y . y$ The differences between the averages indicated in different letters in the same column are statistically significant. 


\begin{tabular}{|c|c|c|c|c|c|}
\hline \multirow[t]{4}{*}{ Habits } & \multicolumn{2}{|c|}{ Permanent teeth } & \multicolumn{2}{|l|}{ Milk teeth } & \multirow[t]{4}{*}{$\mathbf{p}$} \\
\hline & \multicolumn{2}{|l|}{ DMFT $^{c}$} & \multicolumn{2}{|l|}{$\mathrm{dmft}^{\mathrm{d}}$} & \\
\hline & $\mathrm{X} \pm \mathrm{SS}$ & Median & $\mathrm{X} \pm \mathrm{SS}$ & Median & \\
\hline & & (Min-Max) & & (Min-Max) & \\
\hline Never & $\begin{array}{l}2.2 \pm \\
1.90\end{array}$ & $\begin{array}{l}2.00(00- \\
5.00)\end{array}$ & $1.6 \pm 2.22$ & $\begin{array}{l}0.50(00- \\
6.00)\end{array}$ & \\
\hline \multicolumn{5}{|c|}{ The frequency of tooth brushing ${ }^{b}$} & \multirow{7}{*}{$\begin{array}{l}0.392^{c} \\
0.015^{d *} \\
(x-y)\end{array}$} \\
\hline 1 time per day & $\begin{array}{l}2.1 \pm \\
1.94\end{array}$ & $\begin{array}{l}2.00(00- \\
9.00)\end{array}$ & $\begin{array}{l}1.1 \pm \\
1.60^{x y}\end{array}$ & $\begin{array}{l}0.00(00- \\
6.00)\end{array}$ & \\
\hline 2 times a day & $\begin{array}{l}1.8 \pm \\
1.80\end{array}$ & $\begin{array}{l}1.00(00- \\
6.00)\end{array}$ & $\begin{array}{l}0.6 \pm \\
0.92^{x y}\end{array}$ & $\begin{array}{l}0.00(00- \\
4.00)\end{array}$ & \\
\hline 3 times per day & $\begin{array}{l}1.3 \pm \\
1.42\end{array}$ & $\begin{array}{l}1.00(00- \\
4.00)\end{array}$ & $0.0 \pm 0.00^{x}$ & $0.00(00-00)$ & \\
\hline Several times a week & $\begin{array}{l}1.7 \pm \\
1.93\end{array}$ & $\begin{array}{l}1.00(00- \\
5.00)\end{array}$ & $\begin{array}{l}1.1 \pm \\
1.78^{x y}\end{array}$ & $\begin{array}{l}0.00(00- \\
6.00)\end{array}$ & \\
\hline Sometimes & $\begin{array}{l}2.4 \pm \\
2.02\end{array}$ & $\begin{array}{l}2.00(00- \\
9.00)\end{array}$ & $1.5 \pm 1.93^{y}$ & $\begin{array}{l}1.00(00- \\
9.00)\end{array}$ & \\
\hline Never & $\begin{array}{l}2.2 \pm \\
1.81\end{array}$ & $\begin{array}{l}2.00(00- \\
5.00)\end{array}$ & $\begin{array}{l}1.6 \pm \\
2.22^{x y}\end{array}$ & $\begin{array}{l}0.50(00- \\
6.00)\end{array}$ & \\
\hline \multicolumn{2}{|l|}{ Brushing style ${ }^{b}$} & & & & \multirow{4}{*}{$\begin{array}{l}0.111^{c} \\
0.121^{d}\end{array}$} \\
\hline Left to right & $\begin{array}{l}1.9 \pm \\
2.00\end{array}$ & $\begin{array}{l}1.00(00- \\
9.00)\end{array}$ & $1.2 \pm 1.68$ & $\begin{array}{l}0.00(00- \\
6.00)\end{array}$ & \\
\hline Up and down & $\begin{array}{l}2.5 \pm \\
1.87\end{array}$ & $\begin{array}{l}2.00(00- \\
8.00)\end{array}$ & $1.2 \pm 1.69$ & $\begin{array}{l}1.00(00- \\
9.00)\end{array}$ & \\
\hline Circular & $\begin{array}{l}1.8 \pm \\
1.67\end{array}$ & $\begin{array}{l}1.00(00- \\
6.00)\end{array}$ & $0.6 \pm 1.20$ & $\begin{array}{l}0.00(00- \\
5.00)\end{array}$ & \\
\hline \multicolumn{6}{|l|}{ aMann-Whitney U Test } \\
\hline \multicolumn{6}{|l|}{${ }^{\text {b} K r u s k a l ~ W a l l i s ~ T e s t ~}$} \\
\hline \multicolumn{6}{|l|}{${ }^{c} \mathrm{p}$ value for $\mathrm{DMFT}$} \\
\hline \multicolumn{6}{|l|}{${ }^{d} p$ value for $p d m f t$} \\
\hline \multicolumn{6}{|l|}{${ }^{*} p<0.05$} \\
\hline
\end{tabular}




\begin{tabular}{|c|c|c|c|c|c|}
\hline \multirow[t]{4}{*}{ Habits } & \multicolumn{2}{|c|}{ Permanent teeth } & \multicolumn{2}{|l|}{ Milk teeth } & \multirow[t]{4}{*}{$\mathbf{p}$} \\
\hline & \multicolumn{2}{|c|}{$\mathrm{DMFT}^{\mathrm{c}}$} & \multicolumn{2}{|l|}{$\mathrm{dmft}^{\mathrm{d}}$} & \\
\hline & $\mathrm{X} \pm \mathrm{SS}$ & Median & $\mathrm{X} \pm \mathrm{SS}$ & Median & \\
\hline & & (Min-Max) & & (Min-Max) & \\
\hline Do not know & $\begin{array}{l}2.2 \pm \\
3.49\end{array}$ & $\begin{array}{l}1.00(00- \\
9.00)\end{array}$ & $1.2 \pm 1.94$ & $\begin{array}{l}0.50(00- \\
5.00)\end{array}$ & \\
\hline Never & $\begin{array}{l}2.2 \pm \\
1.81\end{array}$ & $\begin{array}{l}2.00(00- \\
5.00)\end{array}$ & $1.6 \pm 2.22$ & $\begin{array}{l}0.50(00- \\
6.00)\end{array}$ & \\
\hline \multicolumn{5}{|l|}{ Brushing duration ${ }^{b}$} & \multirow{6}{*}{$\begin{array}{l}0.502^{c} \\
0.017^{d *} \\
(x-y)\end{array}$} \\
\hline Less than 1 min & $\begin{array}{l}2.3 \pm \\
2.16\end{array}$ & $\begin{array}{l}2.00(00- \\
9.00)\end{array}$ & $\begin{array}{l}1.2 \pm \\
1.85^{x y}\end{array}$ & $\begin{array}{l}0.00(00- \\
9.00)\end{array}$ & \\
\hline $2-3 \min$ & $\begin{array}{l}1.8 \pm \\
1.70\end{array}$ & $\begin{array}{l}1.00(00- \\
6.00)\end{array}$ & $\begin{array}{l}0.8 \pm \\
1.29^{x y}\end{array}$ & $\begin{array}{l}0.00(00- \\
6.00)\end{array}$ & \\
\hline More than $3 \mathrm{~min}$ & $\begin{array}{l}2.3 \pm \\
1.91\end{array}$ & $\begin{array}{l}2.00(00- \\
6.00)\end{array}$ & $0.2 \pm 0.58^{x}$ & $\begin{array}{l}0.00(00- \\
2.00)\end{array}$ & \\
\hline Do not know & $\begin{array}{l}2.3 \pm \\
2.30\end{array}$ & $\begin{array}{l}2.00(00- \\
9.00)\end{array}$ & $1.9 \pm 1.20^{y}$ & $\begin{array}{l}2.00(00- \\
6.00)\end{array}$ & \\
\hline Never & $\begin{array}{l}2.2 \pm \\
1.81\end{array}$ & $\begin{array}{l}2.00(00- \\
5.00)\end{array}$ & $\begin{array}{l}1.6 \pm \\
2.22^{x y}\end{array}$ & $\begin{array}{l}0.50(00- \\
6.00)\end{array}$ & \\
\hline \multicolumn{3}{|c|}{ The frequency of brush replacement ${ }^{b}$} & & & $0.254^{c}$ \\
\hline Quarterly & $\begin{array}{l}1.6 \pm \\
1.82\end{array}$ & $\begin{array}{l}1.00(00- \\
6.00)\end{array}$ & $0.7 \pm 1.37$ & $\begin{array}{l}0.00(00- \\
6.00)\end{array}$ & $0.114^{d}$ \\
\hline Once in a six month & $\begin{array}{l}1.8 \pm \\
1.75\end{array}$ & $\begin{array}{l}1.00(00- \\
6.00)\end{array}$ & $0.8 \pm 1.37$ & $\begin{array}{l}0.00(00- \\
6.00)\end{array}$ & \\
\hline Once a year & $\begin{array}{l}2.3 \pm \\
2.09\end{array}$ & $\begin{array}{l}2.00(00- \\
9.00)\end{array}$ & $1.2 \pm 1.70$ & $\begin{array}{l}1.00(00- \\
9.00)\end{array}$ & \\
\hline \multicolumn{6}{|l|}{ aMann-Whitney U Test } \\
\hline \multicolumn{6}{|l|}{${ }^{b}$ Kruskal Wallis Test } \\
\hline \multicolumn{6}{|l|}{${ }^{c} \mathrm{p}$ value for DMFT } \\
\hline \multicolumn{6}{|l|}{${ }^{d} p$ value for $p d m f t$} \\
\hline \multicolumn{6}{|l|}{${ }^{*} p<0.05$} \\
\hline
\end{tabular}




\begin{tabular}{|c|c|c|c|c|c|}
\hline \multirow[t]{4}{*}{ Habits } & \multicolumn{2}{|c|}{ Permanent teeth } & \multicolumn{2}{|l|}{ Milk teeth } & \multirow[t]{4}{*}{$\mathrm{p}$} \\
\hline & \multicolumn{2}{|c|}{$\mathrm{DMFT}^{\mathrm{c}}$} & \multicolumn{2}{|l|}{$\mathrm{dmft}^{\mathrm{d}}$} & \\
\hline & $\mathrm{X} \pm \mathrm{SS}$ & Median & $\mathrm{X} \pm \mathrm{SS}$ & Median & \\
\hline & & (Min-Max) & & (Min-Max) & \\
\hline Once in two years & $\begin{array}{l}2.4 \pm \\
1.30\end{array}$ & $\begin{array}{l}3.00(00- \\
4.00)\end{array}$ & $1.3 \pm 1.80$ & $\begin{array}{l}0.00(00- \\
5.00)\end{array}$ & \\
\hline Never & $\begin{array}{l}2.4 \pm \\
1.85\end{array}$ & $\begin{array}{l}2.00(00- \\
5.00)\end{array}$ & $1.4 \pm 2.06$ & $\begin{array}{l}0.50(00- \\
6.00)\end{array}$ & \\
\hline \multicolumn{6}{|c|}{ aMann-Whitney U Test } \\
\hline \multicolumn{6}{|l|}{${ }^{\text {b} K r u s k a l ~ W a l l i s ~ T e s t ~}$} \\
\hline \multicolumn{6}{|l|}{${ }^{c} p$ value for DMFT } \\
\hline \multicolumn{6}{|l|}{${ }^{d} p$ value for $p d m f t$} \\
\hline \multicolumn{6}{|l|}{${ }^{*} \mathrm{p}<0.05$} \\
\hline
\end{tabular}

Those consuming crackers, cornflakes, bread, flavored milk, dried fruit, instant fruit juice, fizzy drinks, iced teas, energy drinks, dessert, candy\delight etc., pastry products, jam, table sugar, jelly food and sugary chewing gum have higher mean DMFT values than those who do not consume such foods and drinks, but the differences are not statistically significant $(p>0.05)$. Considering the mean dmfs values, those who do not consume crackers, chips, bread, dried fruit, instant fruit juice, fruity drinks, fizzy drinks, energy drinks, dessert, cookies, cakes, pudding, biscuits, chocolate, table sugar, jelly foods and sugary chewing gum have lower mean dmfs values and the differences are statistically significant only for molasses and table sugar $(p<0.05)$ (Table 4$)$. The mean duration of breastfeeding of children is $14.1 \pm 7.46$ months and the mean time of starting complementary feeding is $6.1 \pm 1.64$ months. It was determined that children with low socioeconomic status have less breastfeeding time $(\mathrm{p}<0.05)$, and oral and dental health indicators do not differ according to breastfeeding time and the time of starting complementary feeding $(p>0.05)$ (data not shown). 
Table 4

Oral health indicators of children according to their nutrient consumption status

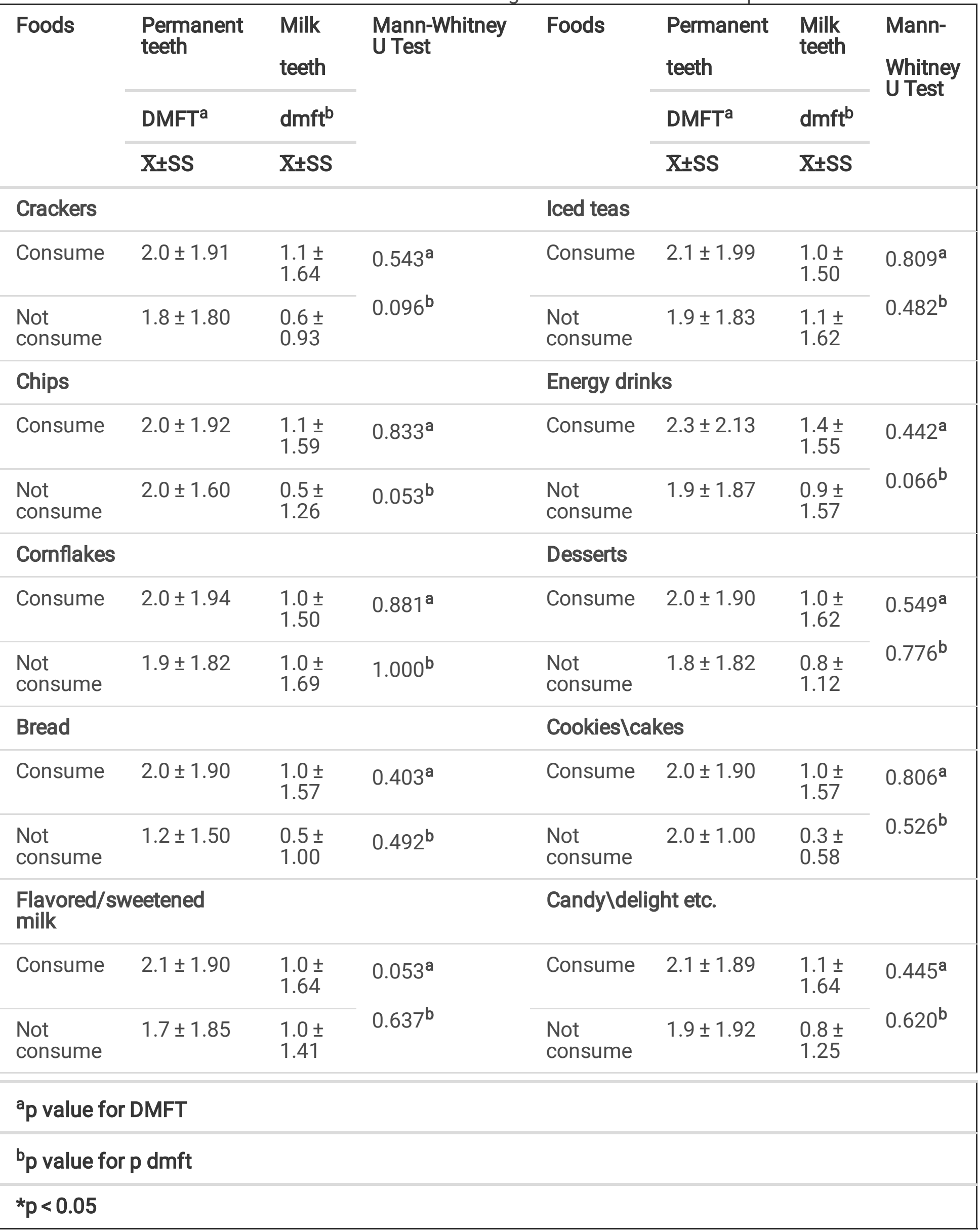




\begin{tabular}{|c|c|c|c|c|c|c|c|}
\hline \multirow[t]{3}{*}{ Foods } & $\begin{array}{l}\text { Permanent } \\
\text { teeth }\end{array}$ & $\begin{array}{l}\text { Milk } \\
\text { teeth }\end{array}$ & \multirow[t]{3}{*}{$\begin{array}{l}\text { Mann-Whitney } \\
\text { U Test }\end{array}$} & \multirow[t]{3}{*}{ Foods } & $\begin{array}{l}\text { Permanent } \\
\text { teeth }\end{array}$ & $\begin{array}{l}\text { Milk } \\
\text { teeth }\end{array}$ & \multirow{3}{*}{$\begin{array}{l}\text { Mann- } \\
\text { Whitney } \\
\text { U Test }\end{array}$} \\
\hline & DMFT $^{\mathrm{a}}$ & $\mathrm{dmft}^{\mathrm{b}}$ & & & DMFT $^{a}$ & $\mathrm{dmft}^{\mathrm{b}}$ & \\
\hline & $\mathrm{X} \pm \mathrm{SS}$ & $\mathrm{X} \pm S S$ & & & $\mathrm{X} \pm \mathrm{SS}$ & $\mathrm{X} \pm$ SS & \\
\hline \multicolumn{4}{|c|}{ Dried Fruits } & \multicolumn{4}{|c|}{ Pastry products (muffin, pastry, fruit pie) } \\
\hline Consume & $2.1 \pm 1.93$ & $\begin{array}{l}1.1 \pm \\
1.64\end{array}$ & \multirow{2}{*}{$\begin{array}{l}0.335^{a} \\
0.681^{b}\end{array}$} & Consume & $2.0 \pm 1.90$ & $\begin{array}{l}1.0 \pm \\
1.60\end{array}$ & \multirow{2}{*}{$\begin{array}{l}0.565^{a} \\
0.639^{b}\end{array}$} \\
\hline $\begin{array}{l}\text { Not } \\
\text { consume }\end{array}$ & $1.8 \pm 1.82$ & $\begin{array}{l}0.9 \pm \\
1.38\end{array}$ & & $\begin{array}{l}\text { Not } \\
\text { consume }\end{array}$ & $1.8 \pm 1.87$ & $\begin{array}{l}0.8 \pm \\
1.0\end{array}$ & \\
\hline \multicolumn{4}{|c|}{ Instant Fruit Juice } & \multicolumn{4}{|l|}{ Honey } \\
\hline Consume & $2.1 \pm 1.95$ & $\begin{array}{l}1.1 \pm \\
1.63\end{array}$ & \multirow{2}{*}{$\begin{array}{l}0.432^{a} \\
0.118^{b}\end{array}$} & Consume & $2.0 \pm 1.90$ & $\begin{array}{l}1.1 \pm \\
1.66\end{array}$ & \multirow{2}{*}{$\begin{array}{l}0.781^{a} \\
0.767^{b}\end{array}$} \\
\hline $\begin{array}{l}\text { Not } \\
\text { consume }\end{array}$ & $1.6 \pm 1.37$ & $\begin{array}{l}0.5 \pm \\
0.73\end{array}$ & & $\begin{array}{l}\text { Not } \\
\text { consume }\end{array}$ & $2.0 \pm 1.87$ & $\begin{array}{l}0.8 \pm \\
1.15\end{array}$ & \\
\hline \multicolumn{4}{|c|}{ Fruity Drinks } & \multicolumn{4}{|l|}{ Molasses } \\
\hline Consume & $2.0 \pm 1.92$ & $\begin{array}{l}1.1 \pm \\
1.64\end{array}$ & \multirow{2}{*}{$\begin{array}{l}0.929^{a} \\
0.667^{b}\end{array}$} & Consume & $2.0 \pm 1.94$ & $\begin{array}{l}1.2 \pm \\
1.72\end{array}$ & \multirow{2}{*}{$\begin{array}{l}0.972^{a} \\
0.006^{b \star}\end{array}$} \\
\hline $\begin{array}{l}\text { Not } \\
\text { consume }\end{array}$ & $2.0 \pm 1.78$ & $\begin{array}{l}0.7 \pm \\
1.08\end{array}$ & & $\begin{array}{l}\text { Not } \\
\text { consume }\end{array}$ & $2.0 \pm 1.80$ & $\begin{array}{l}0.6 \pm \\
1.04\end{array}$ & \\
\hline \multicolumn{4}{|c|}{ Fizzy Drinks } & \multicolumn{4}{|l|}{ Jam } \\
\hline Consume & $2.0 \pm 1.93$ & $\begin{array}{l}1.1 \pm \\
1.66\end{array}$ & \multirow{2}{*}{$\begin{array}{l}0.995^{a} \\
0.620^{b}\end{array}$} & Consume & $2.1 \pm 1.93$ & $\begin{array}{l}1.0 \pm \\
1.54\end{array}$ & \multirow{2}{*}{$\begin{array}{l}0.113^{a} \\
0.855^{b}\end{array}$} \\
\hline $\begin{array}{l}\text { Not } \\
\text { consume }\end{array}$ & $1.9 \pm 1.77$ & $\begin{array}{l}0.8 \pm \\
1.20\end{array}$ & & $\begin{array}{l}\text { Not } \\
\text { consume }\end{array}$ & $1.7 \pm 1.76$ & $\begin{array}{l}0.9 \pm \\
1.64\end{array}$ & \\
\hline \multicolumn{3}{|l|}{ Biscuits } & & \multicolumn{4}{|l|}{$\begin{array}{l}\text { Table } \\
\text { sugar }\end{array}$} \\
\hline Consume & $2.0 \pm 1.92$ & $\begin{array}{l}1.1 \pm \\
1.60\end{array}$ & \multirow{2}{*}{$\begin{array}{l}0.728^{a} \\
0.138^{b}\end{array}$} & Consume & $2.1 \pm 1.94$ & $\begin{array}{l}1.1 \pm \\
1.64\end{array}$ & \multirow{2}{*}{$\begin{array}{l}0.516^{a} \\
0.025^{b *}\end{array}$} \\
\hline $\begin{array}{l}\text { Not } \\
\text { consume }\end{array}$ & $2.1 \pm 1.55$ & $\begin{array}{l}0.3 \pm \\
0.48\end{array}$ & & $\begin{array}{l}\text { Not } \\
\text { consume }\end{array}$ & $1.8 \pm 1.64$ & $\begin{array}{l}0.5 \pm \\
0.97\end{array}$ & \\
\hline \multicolumn{8}{|c|}{${ }^{a} p$ value for DMFT } \\
\hline \multicolumn{8}{|c|}{${ }^{b} p$ value for $p \mathrm{dmft}$} \\
\hline${ }^{*} \mathrm{p}<0.05$ & & & & & & & \\
\hline
\end{tabular}




\begin{tabular}{|c|c|c|c|c|c|c|c|}
\hline \multirow[t]{3}{*}{ Foods } & $\begin{array}{l}\text { Permanent } \\
\text { teeth }\end{array}$ & $\begin{array}{l}\text { Milk } \\
\text { teeth }\end{array}$ & \multirow[t]{3}{*}{$\begin{array}{l}\text { Mann-Whitney } \\
\text { U Test }\end{array}$} & \multirow[t]{3}{*}{ Foods } & $\begin{array}{l}\text { Permanent } \\
\text { teeth }\end{array}$ & $\begin{array}{l}\text { Milk } \\
\text { teeth }\end{array}$ & \multirow{3}{*}{$\begin{array}{l}\text { Mann- } \\
\text { Whitney } \\
\text { U Test }\end{array}$} \\
\hline & DMFT $^{\mathrm{a}}$ & $\mathrm{dmft}^{\mathrm{b}}$ & & & $\mathrm{DMFT}^{\mathrm{a}}$ & $\mathrm{dmft}^{\mathrm{b}}$ & \\
\hline & $\mathrm{X} \pm \mathrm{SS}$ & $\mathrm{X} \pm \mathrm{SS}$ & & & $\mathrm{X} \pm \mathrm{SS}$ & $\mathrm{X} \pm \mathrm{SS}$ & \\
\hline \multicolumn{2}{|l|}{ Chocolate } & & \multirow{3}{*}{$\begin{array}{l}0.614^{a} \\
0.504^{b}\end{array}$} & $\begin{array}{l}\text { Jelly } \\
\text { food }\end{array}$ & & & \\
\hline Consume & $2.0 \pm 1.90$ & $\begin{array}{l}1.0 \pm \\
1.58\end{array}$ & & Consume & $2.3 \pm 2.13$ & $\begin{array}{l}1.3 \pm \\
1.62\end{array}$ & \multirow{3}{*}{$\begin{array}{l}0.410^{\mathrm{a}} \\
0.061^{\mathrm{b}}\end{array}$} \\
\hline $\begin{array}{l}\text { Not } \\
\text { consume }\end{array}$ & $2.2 \pm 1.75$ & $\begin{array}{l}0.5 \pm \\
0.75\end{array}$ & & $\begin{array}{l}\text { Not } \\
\text { consume }\end{array}$ & $1.9 \pm 1.81$ & $\begin{array}{l}0.9 \pm \\
1.54\end{array}$ & \\
\hline \multicolumn{3}{|c|}{ Sugary Chewing Gum } & $0.648^{a}$ & \multicolumn{3}{|l|}{ Pudding } & \\
\hline Consume & $2.1 \pm 1.96$ & $\begin{array}{l}1.1 \pm \\
1.63\end{array}$ & $0.736^{b}$ & Consume & $1,9 \pm 1,90$ & $\begin{array}{l}1,0 \pm \\
1,61\end{array}$ & \multirow{2}{*}{$\begin{array}{l}0,236^{a} \\
0,796^{b}\end{array}$} \\
\hline $\begin{array}{l}\text { Not } \\
\text { consume }\end{array}$ & $1.9 \pm 1.73$ & $\begin{array}{l}0.9 \pm \\
1.39\end{array}$ & & $\begin{array}{l}\text { Not } \\
\text { consume }\end{array}$ & $2,3 \pm 1,87$ & $\begin{array}{l}0,9 \pm \\
1,41\end{array}$ & \\
\hline \multicolumn{8}{|c|}{${ }^{a} p$ value for DMFT } \\
\hline \multicolumn{8}{|c|}{$b_{p}$ value for $p d m f t$} \\
\hline${ }^{*} p<0.05$ & & & & & & & \\
\hline
\end{tabular}

It was discovered that there is a negative relationship between waist-to-height ratio and FT; between waist circumference and FT and DMFT; between hip circumference and DMFT $(p<0.05)$. It was also found that there is a negative relationship between dt and Body Mass Index, hip circumference, upper middle arm circumference, biceps skinfold thickness and triceps skinfold thickness $(\mathrm{p}<0.01)$; and between $\mathrm{ft}$ and hip circumference $(p<0.05)$. There is a negative relationship between $\mathrm{dmft}$ and Body Mass Index, waist circumference, hip circumference, upper middle arm circumference, biceps skinfold thickness and triceps skinfold thickness $(p<0.01)$. It was obtained that socioeconomic status is correlated with DT and DMFT negatively; and with FT positively $(p<0.01)$. Maternal education level was correlated with DT $(p<0.01)$, MT $(p<0.05)$ and DMFT $(p<0.05)$ negatively; and with FT positively $(p<0.05)$. It was also found that there is a negative relationship between paternal education level and DT $(p<0.01)$, MT $(p<0.05)$ and DMFT $(p<0.01)$. It was determined that there is a negative relationship between $\mathrm{dt}$ and $\mathrm{dmft}$ and socioeconomic status, maternal and paternal educational status $(p<0.05)($ Table 5$)$. 
Table 5

Correlation between oral health indicators and children's anthropometric measurements, socioeconomic status and educational status of parents

\begin{tabular}{|c|c|c|c|c|c|c|c|}
\hline \multirow[t]{2}{*}{ Variables } & \multicolumn{4}{|c|}{ Permanent teeth } & \multicolumn{3}{|c|}{ Milk teeth } \\
\hline & DT & MT & FT & DMFT & $\mathrm{dt}$ & $\mathrm{ft}$ & $\mathrm{dmft}$ \\
\hline \multicolumn{8}{|l|}{$\begin{array}{l}\text { Anthropometric } \\
\text { measurements }\end{array}$} \\
\hline \multirow[t]{2}{*}{ Body Mass Index $\left(\mathrm{kg} \backslash \mathrm{m}^{2}\right)$} & -0.065 & -0.026 & -0.055 & -0.087 & -0.227 & -0.087 & -0.245 \\
\hline & 0.305 & 0.677 & 0.387 & 0.169 & $0.000 * \star$ & 0.165 & $0.000 * *$ \\
\hline \multirow[t]{2}{*}{ Waist-to-height ratio } & -0.025 & 0.027 & -0.147 & -0.087 & -0.036 & -0.100 & -0.053 \\
\hline & 0.695 & 0.670 & $0.019 *$ & 0.165 & 0.564 & 0.113 & 0.398 \\
\hline \multirow[t]{2}{*}{ Waist circumference (cm) } & -0.081 & -0.043 & -0.127 & -0.135 & -0.161 & -0.122 & -0.183 \\
\hline & 0.196 & 0.491 & $0.043^{\star}$ & $0.031 *$ & 0.010 & 0.053 & $0.003^{* *}$ \\
\hline \multirow[t]{2}{*}{ Hip circumference $(\mathrm{cm})$} & -0.096 & -0.054 & -0.070 & -0.125 & -0.250 & -0.128 & -0.272 \\
\hline & 0.128 & 0.391 & 0.263 & $0.047 *$ & $0.000 * *$ & $0.042^{*}$ & $0.000 * *$ \\
\hline \multirow{2}{*}{$\begin{array}{l}\text { Upper middle arm } \\
\text { circumference (cm) }\end{array}$} & -0.076 & -0.049 & -0.057 & -0.103 & -0.225 & -0.087 & -0.240 \\
\hline & 0.226 & 0.437 & 0.363 & 0.103 & $0.000 * \star$ & 0.165 & $0.000 * *$ \\
\hline \multirow{2}{*}{$\begin{array}{l}\text { Biceps skinfold thickness } \\
(\mathrm{mm})\end{array}$} & -0.080 & -0.025 & -0.008 & -0.089 & -0.171 & -0.069 & -0.183 \\
\hline & 0.204 & 0.689 & 0.901 & 0.158 & $0.006 * *$ & 0.272 & $0.003^{* *}$ \\
\hline \multirow{2}{*}{$\begin{array}{l}\text { Triceps skinfold thickness } \\
(\mathrm{mm})\end{array}$} & -0.065 & -0.037 & -0.043 & -0.096 & -0.183 & -0.094 & -0.197 \\
\hline & 0.303 & 0.557 & 0.498 & 0.127 & $0.003^{\star *}$ & 0.135 & $0.002^{\star *}$ \\
\hline \multicolumn{8}{|c|}{ Socioeconomic and educational status } \\
\hline \multirow[t]{2}{*}{ Socioeconomic status } & -0.270 & -0.118 & 0.202 & -0.204 & -0.138 & 0.050 & -0.125 \\
\hline & $0.000 * *$ & 0.061 & $0.001 * *$ & $0.001 * *$ & $0.028 *$ & 0.430 & $0.046^{*}$ \\
\hline \multirow{2}{*}{$\begin{array}{l}\text { Maternal education } \\
\text { status }\end{array}$} & -0.215 & -0.139 & 0.135 & -0.172 & -0.142 & 0.049 & -0.127 \\
\hline & $0.001^{\star \star}$ & $0.027 *$ & $0.032^{\star}$ & $0.006^{* *}$ & $0.023^{\star}$ & 0.432 & $0.044^{\star}$ \\
\hline \multirow[t]{2}{*}{ Paternal education status } & -0.220 & -0.148 & 0.117 & -0.176 & -0.158 & 0.105 & -0.142 \\
\hline & $0.000 * \star$ & $0.018^{*}$ & 0.062 & $0.005^{\star \star}$ & $0.011^{*}$ & 0.095 & $0.024^{\star}$ \\
\hline \multicolumn{8}{|l|}{ 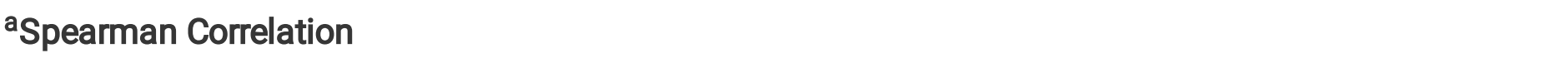 } \\
\hline \multicolumn{8}{|l|}{$*_{p}<0.05$} \\
\hline .01 & & & & & & & \\
\hline
\end{tabular}




\section{Discussion}

The present study was conducted with a total of 254 12-year-old children (112 boys, 142 girls) enrolled in three different middle schools (high, moderate and low socioeconomic status) to examine the effects of socioeconomic status, parental education level, oral and dental health practices of children, dietary habits and anthropometric measurements on oral and dental health.

Oral and dental health in school-age children totally depends on oral hygiene behavior of children and their parents, dietary habits, parental education level, socioeconomic status, regular dental examination, adequate fluoride supplementation, oral microflora, age and other demographic and cultural characteristics (Koposova et al., 2010; Koksal et al., 2011; Bafti et al., 2015). It is stated that families with high socioeconomic status behave more conscious about their children's dental health (Akinci, 2008). In this study, it was shown that factors affecting oral and dental health, such as tooth brushing practices, age and frequency of seeing a dentist and oral and dental health education vary by the one's socioeconomic status. Oral and dental health practices were found to be better in the children of families with high socioeconomic status (Table 2). Similarly, in other studies, children with high socioeconomic status are more likely to see a dentist (Edelstein, 2002; Adeniyi et al., 2016) and to have higher rates of regular brushing (Adekoya-Sofowora et al., 2006). The fact that children from higher-income households have more chances to access to dental care, including a more specific diagnostic assessment and have one or more filled teeth explains the difference in oral and dental health by the ones' socioeconomic status. Higher prevalence of caries in lower socioeconomic status may be due to lack of prevention and treatment services most of the time.

Oral and dental diseases are seen different rates in every society and ages. The World Health Organization and the World Dental Federation (FDI) recommended that DMFT should not be more than 3 for 12 years until 2000, as one of the global goals for oral and dental health (FDI/WHO, 1982). In this study, the mean DMFT value is $2.0 \pm 1.90$ and the recommended goal was reached. Considering certain studies conducted by countries, the mean DMFT values were determined as $4.8 \pm 3.22$ in Bosnia and Herzegovina (Amila et al., 2007), 0.14 in Nigeria, (Adekoya-Sofowora et al., 2006), $3.3 \pm 2.3$ in Russia and $0.5 \pm 0.8$ in Norway (Koposova et al., 2010) and 1.64 in Thailand (Narksawat et al., 2009). Dental caries were determined in $70.9 \%$ of the children in the general sample, $61.6 \%$ of boys and $78.2 \%$ of girls ( $p<$ 0.05) (Table 2). There was no significant difference between gender and the mean DMFT $\backslash d m f t$ value which was found to be $1.9 \pm 2.2$ in 12-year-old children in the Study for Oral and Dental Health Profile of Turkey (Gokalp et al., 2007). In parallel with this study, although there are other studies revealing that the mean DMFT in girls is higher (Koksal et al., 2011; Chakravathy et al., 2012), it was determined in some studies that oral and dental health indicators were similar by gender (Esa and Razak, 2001; Koposova et al., 2010; Adeniyi et al., 2016). It is stated that the prevalence of caries may be higher due to the earlier ages for dentition in girls and the emergence of periodontal problems due to hormonal changes in puberty period (Akinci, 2008). 
Since dental caries has a multifaceted etiology including general health, nutrition, plaque, saliva secretion, type and amount of microorganism, sensitivity of host, oral hygiene habits, use of fluoride, social and behavioral factors, any relationship between oral and dental hygiene practices and caries is difficult to be detected (Karadas et al., 2007). In this study, it was found that the indicators for milk teeth of those who have higher tooth brushing time and frequency are better $(p<0.05)($ Table 3$)$. Proper oral and dental hygiene is also effective in preventing many diseases that are not associated with caries. The most common diseases such as caries and periodontal diseases are caused by poor oral hygiene practices as well as other factors (Ljaljević et al., 2012), children are imported to be educated in subjects such as brushing style, duration, frequency etc.

Dietary habits play an important role in general health status and oral health. In one study, the predominant factor in caries risk profile was shown to be diet (Amila et al., 2007). In this study, the mean DMFT $\backslash d m f t$ values of the children consuming foods with high cariogenic potential were determined likely to be high (Table 4). In a study conducted to examine the effect of backward dietary habits of children on dental health, those who consumed foods increasing the risk of dental caries more than three times a day at the age of one and those who consumed candy more than once a week at the age of three were found to have higher number of decayed and filled teeth at the age of fifteen (Alm et al., 2008). The negative relationship between nutritional status and caries is explained by main meals and snacks. Main meals are stated to contain higher protein and fat and lower sugar than snacks so that snacks are associated with caries. While being exposed to sugary and starchy foods during meals reduces the risk of caries, it was revealed that high sugar consumption with snacks increase such risk (Narksawat et al., 2009).

Dental caries, obesity and malnutrition are global diseases with adverse effects on health (Vázquez-Nava et al., 2010; Weraarchakul and Weraarchakul, 2017). As there are common risk factors for these diseases, the relationship between body weight and tooth decay has been the subject of many studies (Sadeghi and Alizadeh 2007; Loyola-Rodriguez et al., 2011; Kumar et al., 2017; Dikshit et al., 2018). People who have an unbalanced diet with low nutritional value and high sugar and energy content are often affected by both malnutrition and caries. In addition, it is stated that there is a positive relationship between obesity and dental caries with increasing food and refined food consumption and consumption frequency. Therefore, it was investigated whether there is a causal relationship between dental diseases and anthropometric measurements or whether they share the same risk factors (Hafez, 2017). In this study, a negative relationship was found between anthropometric measurements and oral and dental health indicators (Table 5). Contradictory results were found in both research and review studies on body weight and oral health in children. Some studies showed a positive correlation between body weight and tooth decay (Bailleul-Forestier et al., 2007; Alm et al., 2008; Gerdin et al., 2008; Honne et al., 2012), some of them revealed a negative relationship (Koksal et al., 2011; Lueangpiansamut et al., 2012; Bafti et al., 2015), and others found no relationship between them (Costacurta et al., 2011; Adeniyi et al., 2016; Almerich-Torres et al., 2016; Upadhyay et al., 2016). Besides, different results were reached according to different age groups (Narksawat et al., 2009; Kesim et al., 2016). A negative relationship between anthropometric measurements and tooth decay may be caused by the risk of a weak immune system and dietary habits based on foods with low nutritional values and high energy foods in children with low 
body weight. The difficulty in studying the relationship between dental caries and obesity is due to the fact that many factors need to be measured at the same time in a standard way (Almerich-Torres et al., 2016).

Socioeconomic factors have become increasingly scrutinized in studies as they affect the prevalence of dental caries, oral health practices, and parental knowledge on oral and dental health (Popoola et al., 2013; Kato et al., 2017). In this study, it was determined that socioeconomic status and parental education level are related to oral health indicators (Table 5). Similarly, in other studies, socioeconomic status and parental education level were associated with oral health indicators (Adekoya. Sofowora et al., 2006; Adeniyi et al., 2016; Amila et al., 2007; Bafti et al., 2015; Murtomaa, 2011; Koksal et al., 2011). In some studies, no relation was found between parental education level and dental caries (Chakravathy et al., 2012; Lueangpiansamut et al., 2012). It is important that both children and their parents with low socioeconomic level are educated in oral health, awareness raising and guided to make more use of treatment services.

Consequently, it was revealed in this study that dietary habits, anthropometric measurements, oral and dental health practices, gender, parental socioeconomic and education levels are effective on caries risk. It is recommended that children and parents with low socioeconomic status should be given education on oral and dental health practices and guidance to dental care services should be increased. Regulation of dietary habits of children is considerable both for anthropometric measurements and prevention of dental caries. In assessing the effect of dietary habits on dental health, the amount and frequency of consumed foods should be examined in more detail.

\section{Abbreviations}

DT

decayed teeth, MT:missing teeth, FT:filled teeth, DMFT:Decayed, Missing, and Filled Teeth, DMFS:Decayed, Missing, and Filled Surfaces, SPSS:Statistical Package for the Social Sciences, FDI:World Dental Federation.

\section{Declarations}

\section{Acknowledgements}

We are grateful to all those who participated in the questionnaire survey and contributed their valuable thoughts and comments on this study.

\section{Author Contributions}

A.-C.J. and A.Y. contributed to the conception, design, data acquisition, analysis and interpretation, and drafted and critically revised the manuscript; $0 . A-0$. contributed to the conception, design, and 
interpretation and critically revised the manuscript. All authors gave their final approval and agree to be accountable for all aspects of the work.

\section{Funding}

No funding received.

\section{Availability of data and materials}

Please contact the corresponding author for data requests.

\section{Ethics approval and consent to participate}

The research protocol was approved by the Ethics Committee of Mardin Artuklu University dated 11.01.2018 and no. 2018/01-3. All participants provided informed consent.

\section{Consent for publication}

Not applicable.

\section{Competing interests}

The authors declare that they have no competing interests.

\section{Author details}

${ }^{1}$ Mardin Artuklu University Faculty of Health Sciences Department of Nutrition and Dietetics, ${ }^{2}$ Mardin Provincial Health Directorate Nusaybin State Hospital, Dentist, ${ }^{3}$ Ankara University Faculty of Health Sciences Department of Nutrition and Dietetics.

\section{References}

1. Adekoya-Sofowora CA, Nasir WO, Oginni AO, Taiwo M. Dental caries in 12-year-old suburban Nigerian school children. African Health Sciences, 2006;6(3):145-150.

2. Adeniyi AA, Oyapero AO, Ekekezie 00, Braimoh MO. Dental caries and nutritional status of school children in Lagos, Nigeria-a preliminary survey. Journal of the West African College of Surgeons, 2016;6(3):15-38.

3. Akinci Z. Evulation of Oral Dental Health Situation Children at Mix Dentition Period and Their Related Educational Needs. Master's Thesis, Ankara University Institute of Health Sciences,2008.

4. Alm A, Fåhraeus C, Wendt LK, Koch G, Andersson-Gäre Boel, Birkhed D. Body adiposity status in teenagers and snacking habits in early childhood in relation to approximal caries at 15 years of age. International Journal of Paediatric Dentistry, 2008;18(3):189-196. 
5. Almerich-Torres T, Bellot-Arcís C, Almerich-Silla JM. Relationship between caries, body mass index and social class in Spanish children. Gaceta Sanitaria, 2017;31(6):499-504.

6. Amila Z, Sedin K, Maida G. Caries risk assessment in Bosnian children using Cariogram computer model. International Dental Journal, 2007;57(3):177-183.

7. Ashwell M, Hsieh SD. Six reasons why the waist-to-height ratio is a rapid and effective global indicator for health risks of obesity and how its use could simplify the international public health message on obesity. International Journal of Food Sciences and Nutrition, 2005;56(5):303-307.

8. Bafti LS, Hashemipour MA, Poureslami H, Hoseinian Z. Relationship between body mass index and tooth decay in a population of 3-6-year-old children in Iran. International Journal of Dentistry, 2015;1-5.

9. Bailleul-Forestier, I, Lopes K, Souames M, Azoguy-Levy S, Frelut ML, Boy-Lefevre ML. Caries experience in a severely obese adolescent population. International Journal of Paediatric Dentistry, 2007;17(5):358-363.

10. Chakravathy PK, Chenna D, Chenna V. Association of anthropometric measures and dental caries among a group of adolescent cadets of Udupi district, South India. European Archives of Paediatric Dentistry, 2012;13(5):256-260.

11. Cinar AB, Murtomaa H. Interrelation between obesity, oral health and life-style factors among Turkish school children. Clinical Oral Investigations, 2011;15(2):177-184.

12. Costacurta M, Direnzo L, Bianchi A, Fabiocchi F, De Lorenzo A, Docimo R. Obesity and dental caries in paediatric patients. A cross-sectional study. European Journal of Paediatric Dentistry, 2011;12(2):112-116.

13. Cruz ML, Goran MI. The metabolic syndrome in children and adolescents. Current Diabetes Reports, 2004;4(1):53-62.

14. Dikshit P, Limbu S, Bhattarai R. Relationship of body mass index with dental caries among children attending pediatric dental department in an institute. Journal of the Nepal Medical Association, 2018;56(210):582-586.

15. Edelstein BL. Disparities in oral health and access to care: Findings of national surveys. Ambulatory Pediatrics, 2002;2(2):141-147.

16. Esa R, Razak IA. Dental fluorosis and caries status among 12-13 year-old schoolchildren in Klang district, Malaysia. Annals of Dentistry University of Malaya, 2018;8(1): 20-24.

17. Federation Dentaire Internationale/World Health Organization (FDIIWHO). Global goals for oral health in the year 2000. Int Dent $J, 1982 ; 32(1): 74-7$.

18. Gerdin EW, Angbratt M, Aronsson K, Eriksson E, Johansson I. Dental caries and body mass index by socio-economic status in Swedish children. Community Dentistry and Oral Epidemiology, 2008;36(5):459-465.

19. Gokalp S, Dogan B, Tekcicek M, Berberoglu A and Unluer S. The Oral Health Profile of 5, 12 and 15 Year Olds, Turkey-2004. Journal of Hacettepe Faculty of Dentistry, 2007;31(4): 3-10. 
20. Hafez S. The association of dental caries and periapical lesions with anthropometric measurements in postpartum women in Mangochi, Malawi. Master's Thesis University of Tampere School of Health Sciences, 2017.

21. Honne T, Pentapati K, Kumar N, Acharya S. Relationship between obesity/overweight status, sugar consumption and dental caries among adolescents in South India. International Journal of Dental Hygiene, 2012;10(4):240-244.

22. Karadas M, Tahan E, Köse O, Demirbuğa S. Evaluation of Relationship Between DMFT Index and Oral Hygiene with Tooth Brushing Frequency in 13-20 Year-Old Individuals. Turkiye Klinikleri Journal of Dental Sciences, 2014; 20(3): 177 - 81.

23. Kato H, Tanaka K, Shimizu K, Nagata C, Furukawa S, Arakawa M, Miyake Y. Parental occupations, educational levels, and income and prevalence of dental caries in 3-year-old Japanese children. Environmental Health and Preventive Medicine, 2017;22(80):1-7.

24. Kesim S, Cicek B, Aral Ca, Ozturk A, Mazicioglu MM, Kurtoglu S. Oral health, obesity status and nutritional habits in Turkish children and adolescents: an epidemiological study. Balkan Medical Journal, 2016;33(2):164-172.

25. Koksal E, Tekcicek M, Yalcin SS, Tugrul B, Yalcin S, Pekcan G. Association between anthropometric measurements and dental caries in Turkish schoolchildren. Central European Journal of Public Health, 2011;19(3):147-151.

26. Koposova N,Widstrom E, Eisemann M, Koposov R, Eriksen HM. Oral health and quality of life in Norwegian and Russian schoolchildren: A pilot study. Stomatologija, 2010;12(1):10-16.

27. Kumar S, Kroon J, Lalloo R, Kulkarni S, Johnson NW. Relationship between body mass index and dental caries in children, and the influence of socio-economic status. International Dental Journal, 2017;67(2):91-97.

28. Ljaljević A, Matijević S, Terzić N, Anđelić J, Mugoša B. Significance of proper oral hygiene for health condition of mouth and teeth. Vojnosanitetski Pregled, 2012;69(1): 16-21.

29. Loyola-Rodriguez JP, VILLA-Chavez C, Patiño-Marin N, Aradillas-Garcia C, Gonzalez C, De La CruzMendoza E. Association between caries, obesity and insulin resistance in Mexican adolescents. Journal of Clinical Pediatric Dentistry, 2011;36(1):49-54.

30. Lueangpiansamut J, Chatrchaiwiwatana S, Muktabhant B, Inthalohit W. Relationship between dental caries status, nutritional status, snack foods, and sugar-sweetened beverages consumption among primaryschoolchildren grade 4-6 in Nongbua Khamsaen school, Na Klang district, Nongbua Lampoo Province, Thailand. J Med Assoc Thai, 2012;95(8):1090-7.

31. Moynihan P, Petersen PE. Diet, nutrition and the prevention of dental diseases. Public Health Nutrition, 2004;7(1a):201-226.

32. Narksawat $\mathrm{K}$, Tonmukayakul U, Boonthum A. Association between nutritional status and dental caries in permanent dentition among primary schoolchildren aged 12-14 years, Thailand. South East Asian Journal of Tropical Medicine and Public Health, 2009;40(2):338-344. 
33. Popoola BO, Denloye OO, lyun OI. Influence of parental socioeconomic status on caries prevalence among children seen at the university college hospital, Ibadan. Annals of Ibadan Postgraduate Medicine, 2013;11(2):81-86.

34. Sadeghi M, Alizadeh F. Association between dental caries and body mass index-for-age among 6-11year-old children in Isfahan in 2007. Journal of Dental Research, Dental Clinics, Dental Prospects, 2007;1(3):119-124.

35. Touger-Decker R, Mobley C. Position of the Academy of Nutrition and Dietetics: oral health and nutrition. Journal of the Academy of Nutrition and Dietetics, 2013;113(5):693-701.

36. Upadhyay, S. Srii, R. Srivastava, S. Karki, S. Relationship of early childhood caries and body mass index in children attending a tertiary healthcare center of Nepal. International Journal of Scientific Research, 2016;5(12):12-14.

37. Vázquez-Nava F, Vázquez-Rodríguez EM, Saldívar-González AH, Lin-Ochoa D, Martínez-Perales GM, Joffre-Velázquez VM. Association between obesity and dental caries in a group of preschool children in Mexico. Journal of Public Health Dentistry, 2010;70(2):124-130.

38. Weraarchakul W, Weraarchakul W. Relationship between nutritional status and dental caries in elementary students, Samliam Municipal School, Khon Kaen Province, Thailand. Journal of The Medical Association of Thailand, 2017;100(8):232-239.

39. World Health Organization (WHO). Oral Health Surveys Basic Methods (5th Edition). WHO Library Cataloguing, 2013;1-137.

40. World Health Organization (WHO). WHO Multicentre Growth Reference Study Group. Growth reference data for 5-19 years. 2007. 